\title{
Modifications des fermentations dans le rumen et des niveaux de l'insulinémie induites par une ingestion séparée du complément azoté et du fourrage chez la vache tarie : résultats préliminaires
}

\author{
D Dehareng, JM Godeau, JF Beckers, F Beaussart, W Ndibualonji \\ Laboratoire de Biochimie Normale et Pathologique, Centre de Recherches de I'IRSIA, Faculté de \\ Médecine Vétérinaire, rue des Vétérinaires, 45, B-1070 Bruxelles, Belgique
}

Les effets d'une ingestion séparée du foin et d'un aliment concentré sur les fermentations ruminales et sur les niveaux de l'insulinémie ont été étudiés chez une vache tarie et canulée (rumen). Celle-ci a reçu du foin (F) $(1,3 \mathrm{~kg} / \mathrm{j} ; 12 \mathrm{~g}$ d'azote $(\mathrm{N}) / \mathrm{kg})$ et un aliment concentré (C) $(3 \mathrm{~kg} / \mathrm{j} ; 21,7 \mathrm{~g} \mathrm{~N} / \mathrm{kg})$ selon 4 modes de distribution: $\mathrm{C}$ a été donné $2 \mathrm{~h}$ ou $25 \mathrm{~min}$ avant $\mathrm{F}$ ou encore 25 min ou $2 h$ après $F$. Dans chaque cas, du liquide du rumen a été prélevé en continu (24 $\mathrm{h}$; mesure du $\mathrm{NH}_{3}$ ) et ponctuellement (dosage des acides gras volatils (AGV)). Simultanément, une collecte continue $(24 \mathrm{~h})$ de sang veineux a été réalisée (dosage de l'insuline). Le taux de renouvellement $k$ des ingesta solides a été déterminé $\left(\mathrm{Cr}_{2} \mathrm{O}_{3}\right)$.

L'ingestion de $\mathrm{F} 25 \mathrm{~min}$ avant $\mathrm{C}$, comparativement à l'ingestion de $F 25 \mathrm{~min}$ après $\mathrm{C}, \mathrm{a}$ induit une dégradation plus intense de $C$ parce que retenu dans le rumen ( $k: 2,40$ vs $3,43 \% / h$ : ceci est confirmé par un accroissement quantitatif $(P<0,05)$ du $\mathrm{NH}_{3}(2,40$ vs $1,50 \mathrm{~g} / \mathrm{j})$ et des AGV $(2,82$ vs $2,23 \mathrm{eq} / \mathrm{j})$ dans le rumen, et de l'insuline sanguine $(10,4$ vs $5,7 \mu \mathrm{Ul} / \mathrm{ml})$. Ces résultats confirment les observations de Ruckebusch et al (1981) selon lesquelles l'ingestion en premier lieu d'un aliment convoité comme $\mathrm{C}$ se traduit par son évacuation rapide au travers de l'orifice reticulo-omasal. Par contre, l'ingestion de $\mathrm{F} 2 \mathrm{~h}$ avant $\mathrm{C}$, par rapport $\mathrm{a} 2 \mathrm{~h}$ après $\mathrm{C}$, $a$ induit une dégradation moins intense des aliments comme l'indiquent les taux $k$ $(4,77$ vs $1,83 \% / h)$ et les teneurs ruminales $(P<0,05)$ en $\mathrm{NH}_{3}(1,33$ vs $2,41 \mathrm{~g} / \mathrm{j})$ et en AGV $(1,39$ vs 2,38 eq/j). Néanmoins, ce régime a également favorisé les niveaux de l'insulinémie $(16,6$ vs $4,6 \mu \mathrm{Ul} / \mathrm{ml} ; P<$ $0,05)$.

En conclusion et relativement à la situation inverse, l'ingestion de $\mathrm{F}$ avant $\mathrm{C}$ a eu des effets divergents sur les fermentations du rumen. Lorsque les 2 aliments ont été donnés à $25 \mathrm{~min}$ d'intervalle, le transit de $\mathrm{C}$ a été retardé et la dégradation du $\mathrm{N}$ alimentaire de même que la fermentation des hydrates de carbone ont été favorisées. Par contre, lorsque 2 h ont séparé la distribution des 2 aliments, les évolutions inverses de ces phénomènes ont été observées. Toutefois, l'ingestion de $\mathrm{F}$ avant $\mathrm{C}$ a toujours relevé les niveaux de l'insulinémie, quelle que soit la durée de l'intervalle séparant la distribution des 2 aliments. Une confirmation par un plus grand nombre d'animaux s'avère nécessaire.

Ruckebusch Y, Buéno L, Fioramonti J (1981) In: La Mécanique Digestive Chez les Mammifères. Masson, Paris 